\title{
MEDIEN, PRAKTIKEN UND FORMEN DER IMAGINATION VON HEIMAT IN STEFANIE ZWEIGS EIN MUND VOLL ERDE
}

\author{
Rafatou Tchagao ${ }^{1}$
}

\begin{abstract}
This article deals with the relationship between home and foreign countries using the novel A Mouth Full of Earth by the successful German-Jewish writer Stefanie Zweig. It attempts to grasp the complexity of the construction of home and foreign countries. The analysis focuses on literary descriptive practices, forms, and media, whereby the various concepts of home are encoded in the author's narrative text, which is strongly autobiographically colored. In the following, the concept of home will be examined specifically in the case of the characters father and daughter, since both appear in the novel not only as main characters but also as counter-characters, and thus each has his or her own image of the concept of "home". I mainly pursue the following questions: What does home represent for the daughter figure and what does home signify for the father figure? What are the backgrounds for these differences in the perception of home?
\end{abstract}

Keywords: Stefanie Zweig; Homeland; Foreigner; Exile; Africa.

Zusammenfassung: Der vorliegende Beitrag setzt sich mit dem Verhältnis von Heimat und Fremde auseinander anhand des Romans Ein Mund voll Erde der deutsch-jüdischen Erfolgsschriftstellerin Stefanie Zweig. Dabei wird versucht, die Komplexität von Heimat- und Fremdkonstruktionen zu erfassen. Im Mittelpunkt der Analyse stehen die literarischen Beschreibungspraktiken, Formen und Medien, wodurch die verschiedenen Heimatentwürfe im stark autobiographisch gefärbten narrativen Text der Autorin codiert sind. Konkret wird im Folgenden die Heimatkonzeption bei den Figuren Vater und Tochter untersucht, denn beide erscheinen im Roman nicht nur als Hauptfiguren, sondern auch als Gegenfiguren und haben dadurch jeder ein eigenes Bild für das Konzept „Heimat“. Hauptsächlich gehe ich folgenden Fragenstellungen nach: Was repräsentiert Heimat für die Tochter-Figur und was signifiziert Heimat für die Vater-Figur? Welches sind die Hintergründe für diese Wahrnehmungsunterschiede von Heimat?

Schlüsselwörter: Stefanie Zweig; Heimat; Fremde; Exil; Afrika.

1 PhD in Literaturwissenschaft an der Universität zu Köln, Deutschland. 


\title{
1. FIGUREN IN EIN MUND VOLL ERDE
}

Die Figuren Owuor (Lieblingsfigur) ${ }^{2}$, Jogona, Kinghorn mit dem Spitznamen „Bwana Simba" (S. 167) und vor allem die Tochter- und Vater-Figur gelten als wichtige Figuren für die Bestseller-Autorin. ${ }^{3}$ Dennoch möchte ich mich hier auf die Figuren von Vater und Tochter ${ }^{4}$ beschränken. Dieses Vorgehen beruht auf der Tatsache, dass beide im Laufe des Erzählten als Gegenfiguren gestaltet sind: Einerseits haben beide Figuren keine gemeinsame Vorstellung weder von der Heimat noch von der Fremde; andrerseits sprechen sie - wie es im Folgenden zu zeigen ist - keine gemeinsame Sprache.

\section{1. „NO COMMON MOTHER TONGUE“: TOCHTER VERSUS VATER}

Dass Afrikaner ihre eigene Kultur wie auch jedes andere Volk haben, erfährt der Leser vorwiegend in Ein Mund voll Erde. So rufen z.B. Afrikaner ihrer Kultur gemäß alte Leute nicht unmittelbar mit ihren Namen, sondern nennen sie aus Höflichkeit ,Papa' oder ,Mama', obwohl diese nicht unbedingt ihre genetischen Eltern sind. Während die junge Protagonistin diese kulturelle Besonderheit wahrnimmt, interessiert sich ihr Vater kaum dafür und verbietet ihr bei einem Gespräch, ihn als „Papa“ anzusprechen. Nur damit die afrikanischen Kinder ihn nicht auch „Papa“ nennen, wie es seine Tochter tut. Stattdessen ist ihm der kolonial konnotierte Spitzname aller weißen Männer auf der Farm lieber „Bwana“ (S. 83):

\author{
„Du sollst mich nicht immer Bwana nennen.“ \\ „Nein, Bwana“ $[. .$. \\ „ich werde dich immer Papa nennen. Soll Jogona auch Papa zu dir sagen?“ \\ „Um Himmels willen nein! Dann sag schon Bwana zu mir“ (S. 64).
}

Die konträre Denkweise zwischen Vater und Tochter über die Fremde erfährt der Leser dadurch, dass Vivian sich glücklich fühlt, wenn sie in der Nähe von anderen Figuren, wie der von de Bruin ist und besonders wenn sie als „Kikuyukind“ (S. 86) oder „Burenkind“ (S. 150) wahrgenommen wird. Alle wissen also, dass Vivian in Kenia gut integriert ist, sogar der Bure, der Vivian das erste Mal sieht (vgl. S. 86), aber der Vater nicht (vgl. S. 86); vielleicht weiß er auch bereits, will es jedoch nicht anerkennen. So überlegt sich Vivian, „was

2 Vgl. Schestokat, Karin U.: Memories of Africa: Stefanie Zweig’s Autobiographical Works, S. 60.

3 AKTUELL KULTUR: „Bestseller-Autorin Stefanie Zweig gestorben“. In: https://www.dw.com/de/ bestseller-autorin-stefanie-zweig-gestorben/a-17594522, veröffentlicht am 27.04.2014, abgerufen am 13.07.2020.

4 Diese Beschränkung liegt auch in der Tatsache, dass es keine Mutter-Figur im Roman gibt, was auch die enge Beziehung der Ich-Erzählerin zu ihrem Vater verdeutlicht. Stattdessen wird die Figur von Hanna gestaltet, mit welcher der Vater eine kurze Liebesaffäre gehabt haben soll (vgl. S. 89f.): „Einmal hatte sie sogar gehört, wie er jemandem sagte: Vivian hat ihre Mutter verloren. Dabei hatte Vivian ihre Mutter nie gekannt. Die Mutter war bei ihrer Geburt gestorben, und Vivian fand, da könnte man nicht gut von verlieren sprechen“ (ebd., S. 65). Während die Mutterfigur hier „[...] totally absent“ ist (S. 57), taucht der Vater als eine Figur auf, die vieles in Afrika ignoriert, aber während der ganzen Geschichte ehrlich bleibt: „[...] Er weiß vieles nicht, aber er lügt nicht“" (S. 131). 
ihr Vater wohl dazu sagen würde“ (S. 151), wenn er erfährt, dass sie sich glücklich fühle, als sie für ein afrikanisches Kind gehalten wird. In der Tat zeigt sich Vivian glücklicher in der Nähe von anderen Figuren, die die gleiche Wahrnehmung von der Farm, deren Leuten, deren Umwelt und Alltagsleben wie sie haben. Dies ist nicht so bei ihrem Vater, ihrem Gegenbild. So wünscht sie sich einen Vater wie den Buren Louis de Bruin, der sich auch in Kenia, auf der Farm zu Hause fühlt (vgl. S. 83): „[...] aber de Bruin gefiel ihr. Er sagte Dinge wie sie Jogona sagte, und er sprach nicht wie ihr Vater. Sie rieb ihr Gesicht vorsichtig an seiner Jacke [...]“ (S. 86).

So ähneln Vivians Vater, die vermeintliche Gefährtin Hanna (S. 89f.) und Dixon in ihrer Konzeption einander, insbesondere was ihr Afrikabild betrifft: „Alles, was mit Afrika zusammenhing, interessierte ihn nicht [...]“ (S. 145), denn Kenia gilt für ihn nur als „ein verfluchtes Land“ (S. 145). Dabei ist die Tochter eher dem ehemaligen britischen Jäger ähnlich (vgl. u.a. S. 171), der „vor fünfzig Jahren den afrikanischen Boden betreten hatte“ (S. 167) und sich entschlossen hat, sein ganzes Leben in Afrika zu verbringen, statt mit dem Geld, das sein Vater ihm geschickt hatte, nach England zurück zu kehren, wie es dieser wollte (vgl. S. 168). Vivian geht weiter in ihrer Integration in Afrika und ist nun im Stande, die Afrikaner zu riechen und sie durch ihren jeweiligen Geruch voneinander zu unterscheiden: „Das war Morenu [...]. Ich hab’ ihn gerochen“ (S. 74); doch der Vater hält dies nur für eine Behauptung:

„[...] sie könne Menschen riechen. Sie tat, als sei das so selbstverständlich wie einen Krug Kaffee aus der Küche zu holen. Du willst doch nicht behaupten, dass du die Menschen auf der Farm am Geruch unterscheiden kannst. Für mich haben sie alle die gleichen Gesichter" (S. 75).

Diese ,neutrale Reaktion des Vaters gilt als Symbol der Interesselosigkeit an den Kenianern, deren Sitten und Gebräuche. Denn für ihn sei seine Heimat, Deutschland, der Drehpunkt von allem (vgl. S. 175; S. 190). So erinnert er an das lyrische Ich im Brechts Gedicht ${ }^{5}$. Dazu nähert der Vater hier der Mutter-Figur, Jettel in Nirgendwo in Afrika, die nach Schestokat, wie „a fish out of water“6 konfiguriert wird. Tatsächlich mag der Vater der Protagonistin keine lokale Sprache, genauso wenig wie Jettel'; nun aber können die Farmer kein Deutsch. Deshalb ist er meistens einsam, bis der Bure ihn besucht, der Deutsch kann (vgl. S. 89). Doch mit dem Engländer, Bwana Simba, der kein Deutsch, sondern nur Suaheli und Englisch kann (vgl. S. 169), hat der Vater keine andere Wahl, als Suaheli zu sprechen. Aber mit der Zeit, bei seinem wochenlangen Besuch lässt er sich von Kinghorn die englische Sprache beibringen. So freut sich der Vater, kein Suaheli mehr mit ihm reden

5 Brecht, Bertolt: Über die Bezeichnung Emigranten. In: Jan Knopf (Hrsg.): Lebenskunst in finsteren Zeiten, S. 404.

6 Schestokat, Karin U.: Memories of Africa: Stefanie Zweig's Autobiographical Works, S. 57. Obgleich Vivians Vater hier nicht unmittelbar auftaucht als eine Person, die in Deutschland „oppressed and persecuted by the Nazis“ ist, tritt er nun in einer abstrahierten Weise - in Afrika als der weiße Mann auf, der als „oppressor who obviously victimizes the Africans“ vorgestellt wird (S. 56f.).

7 „She does not speak Swahili [...]“, ebd., S. 57. 
zu müssen; was auch implizit heißt, dass er zwar English nicht mag, dieses aber dennoch dem Suaheli vorzieht: „Von Bwana Simba lernte der Vater Englisch, und die beiden Männer brauchten sich nicht länger nur auf Suaheli zu unterhalten“" (S. 171).

Dass Vivian sich schnell auf Kenia einlässt, ist kein Zufall. Dies liegt am „Anpassungsverhalten von Kindern“" ${ }^{8}$ Denn bei der Problematisierung des Heimatkonzepts spielt insbesondere das Alter eine bedeutende Rolle. So findet Vivian als Kind (wie auch viele exilierte Kinder in den ehemaligen englischen Kolonien) ihre Heimat nicht nur in Kenia, sondern vielmehr auch in der englischen Sprache: „The conceptions of 'homeland' and mother tongue were evidently deeply interconnected for many Jewish refugee children. For some, the colonies where they found safety became home, and English, their mother tongue [...]". Insofern wird die Differenz zwischen europäischer und afrikanischer Kultur eng verschränkt mit der Differenz zwischen den Generationen. Die Anpassung an Kultur, Klima und Sprache(n) des Exillands „was a difficult experience for adult refugees; the response of children was no less complex but ultimately different. " ${ }^{10}$ Während es Vivian leicht fällt, sich der kenianischen Kultur anzupassen, oder, um es mit Reeves Worten zum Ausdruck zu bringen, obgleich sie mit „the speed and eagerness of a child“11 die lokalen Sprachen und Kultur lernt, hat der Vater es schwer und vermisst daher sehr seine Heimat: „As easy as it seems for the child to adjust and adapt to the daily routine in Kenya. As difficult and problematic the same task is [...]"12 für den Vater.

Die schnelle Integration Vivians lässt sich mehrfach feststellen: Zum einen auf der kommunikativen Ebene (dies gilt sowohl für Menschen als auch für Natur und Tiere), zum anderen auf der kulturellen Ebene. So weiß Vivian, bereits am Anfang des Romans, kurz nach ihrer Ankunft in Kenia, durch ihren Jugendfreund Jogona, dass der spirituelle Ausdruck "Hakiri ja Mungu“ eine Beschwörung ist, die demjenigen den Tod bringen könnte, der lügt (S. 60f.): „Das weiß ich. [...] Ich sterbe nicht, ich sage die Wahrheit. Du bist mein Rafiki“ (S. 61). Darüber hinaus weiß sie viel über afrikanische Männer und Frauen wie auch über deren Lebensarten: „Erst als Vivian vor dem Haus stand, wurde ihr bewusst, dass sie nicht neben, sondern hinter Jogona gelaufen war. Wie die Frauen Afrikas, die hinter ihren Männern hergingen“ (S. 200). Auch schreckt die Protagonistin vor nichts bei ihrem Kulturerwerb zurück. So entdeckt sie den afrikanischen Zauber bei allen Stämmen wie z.B. bei den Nandi, wo sie

das Blut einer frisch geschlachteten Gazelle zu trinken [lernte], das Männer stark

\footnotetext{
8 Emre, Merle: Grenz(über)gänge, S. 42. Diesen psychischen Mechanismus bei den Kindern hebt Zweig in fast allen ihren autobiographischen Romanen hervor. So heißt es in Nirgendwo in Afrika: „Kinder finden sich schnell ab. [...] sagte Jettel an dem Tag, als Regina erzählte, sie habe Jaluo gelernt, um mit Owuor und Aja in ihrer Sprache reden zu können [...]" (Zweig, Stefanie: Nirgendwo in Afrika, S. 35). So schafft Vivians kleiner Bruder, der fünfjährige Max, der fast gleiches Schicksal wie seine Schwester nun hat, sich nach der Rückkehr in Deutschland schneller zu integrieren als sie selbst: „Mein kleiner Bruder hatte es noch leichter, sich in Frankfurt einzuleben“ (Vivian, S. 17).
}

9 Reeve, Jennifer: 'No common mother tongue or fatherland', S. 114.

10 Ebd.

11 Ebd.

12 Schestokat, Karin U.: Memories of Africa: Stefanie Zweig's Autobiographical Works, S. 56. 
und Frauen fruchtbar machte. Sie lernte auch die Worte, um einen Mann so verzaubern, dass er keine Augen mehr für andere Frauen hat. Sie wusste, wie ein Kind zu sagen hat, wenn es ein Mann wird, und sie nahm sich in Acht, dass ihr Schatten nie auf eine schwangere Frau fiel, denn ein Schatten ließ das Kind sterben. (S. 173)

Da Vivian vieles über die „Geheimnisse und Zaubersprüche“ der Nandi weiß (S. 180), scheint sie sich sogar einheimischer und damit geborgener in Kenia zu fühlen als Jogona ${ }^{13}$, dem es, im Unterschied zu ihr, gar nicht gelingt, wie die Nandi frisches Blut zu trinken (vgl. Auch S. 187). Und diesen kulturellen Unterschied genießt Vivian triumphierend: „Jogona fragte oft nach den Dingen, die Vivian bei den Nandi lernte, aber sie schwieg über ihre Erlebnisse [...]" (S. 173). Auch als Vivian und Jogona auf dem Weg nach dem Muchau feststellen, dass keiner vor beiden „den Weg gegangen war“ (vgl. S. 181), zeigt sich Vivian neugierig und will trotzdem zu dem kenianischen Medizinmann. Ebenso versteht Vivian die afrikanischen Tiere in ihrer Kommunikation:

Sie wäre bis zur Dunkelheit im Maismehl geblieben, um auf das Heulen der Hyänen zu warten. [...] Sie wartete auf das Echo und hörte gleichzeitig den dumpfen Klang der Trommeln.

„Hörst du die Ngoma?“ [...]

„Natürlich weiß ich, was sie sagen. Sie sagen, dass ein Leopard jagt“ (S. 56).

Darüber hinaus erkennt und preist der Englischlehrer Dixon Vivians sprachliche Leistungen analog zu anderen Mitschülern. So pflegt er andere Kinder daran zu erinnern, dass Vivian außer Deutsch und Englisch auch Suaheli und Kikuyu, also insgesamt vier Sprachen, kann (vgl. S. 142) ${ }^{14}$ : „Sie hat zwei Sprachen gelernt, und ihr könnt noch nicht einmal eine einzige richtig" (S. 142). Auch lernt Vivian mit Hilfe de Bruins jüngster Tochter, Anna, „Afrikaans [...], das dem Deutschen ähnelte“ (S. 139; S. 150). Durch ihren Spracherwerb und Kulturerwerb kennt Vivian nicht nur eines der meistgebrauchten Wörter in Suaheli (z.B. „Kessu“, S. 62) und das beliebte Spiel auf der Farm, nämlich Fragen zu stellen, obwohl man die Antworten kennt (vgl. u.a. S. 102), sondern auch, dass der bildhafte Ausdruck, auf seinen Augen schlafen', auf Suaheli eine Trope für ,Dinge nicht genau genug betrachten' ist $\left(\right.$ vgl. S. 104) ${ }^{15}$. Auch in der strengen britischen Schule in Nakuru, mit „festgesetztem Rhyth-

13 Denn sie weiß - im Gegensatz zu Jogona, (dem territorialen Mensch im Sinne von Greverus), für den es immer nur um die Farm Ol' Joro Orok geht, fast alles über die vielfältigen Stämme des Lands: Chamba- Kikuyu-, Nandi-, Lumbwaleute, (vgl. jeweils S. 80; S. 97f.): „Ich weiß alles von den Nandi“, teilt Vivian z.B. triumphierend dem Kikuyujungen, Jogona (S. 81).

14 Dazu schreibt etwa Karin U. Shestokat: „[T]he white child Regina on the farm, who is Vivian in Ein Mund voll Erde, identifies with Africans and learns both Kikuyu and Nandi on top of Swahili“ (Shestokat, Karin U.: Memories of Africa: Stefanie Zweig's Autobiographical Works, S. 55).

15 In diesem Zusammenhang schrieb bereits etwa Shestokat, Karin U.: „The child Regina (also Vivian) tries to teach this way of communicating to her father, but he usually does not understand, he does not get it“" (ebd., S. 55). 
mus und genau festgelegten Regeln“ (S. 135), wo die Protagonistin weniger glücklich als auf der Farm ist, zeigt sich ihre rasche Integration:

Vivian war in ihrem Leben noch nie geschlagen worden, und sie hatte auch nie erlebt, dass andere Kinder geschlagen worden, aber sie gewöhnte sich schnell an die Strafen. Sie empfand jedenfalls das Brennen der Schläge auf der Haut weit weniger unangenehm als die Traurigkeit, die in ihr war, weil sie eine nie zu stillende Sehnsucht nach der Farm hatte. (S. 137)

Dabei wird Deutsch „zu einer Sprache au second degré" ${ }^{16}$, während Suaheli sich als die Sprache au premier degré erweist; da das schreibende Ich durch sein Alter Ego Vivian ein besonderes Gewicht auf die Rhetorizität und Bildhaftigkeit des Suahelis im Schreibakt legt: „In Ol' Joro Orok, der kleinen Ortschaft, in der die Farm lag, sagte man gerne Dinge, die bekannt waren“ (S. 51). Wobei dies auch die Art und Weise verrät, wie die Handlung erzählt und beschrieben wird. Sie ist tatsächlich von zahlreichen Wiederholungen geprägt und auf der syntaktischen und semantischen Ebene stark gemäß den grammatischen Strukturen und parabolischen Ausdrücken der lokalen Sprache(n) v.a. des Suahelis geschrieben. Mittels einer „tropenreiche[n] und rhetorische[n] Sprache“ des Suahelis ${ }^{17}$ sind also metaphorische Redewendungen und Übersetzungen gemeint, wie ,ein Loch im Kopf haben', d.h. ,krank

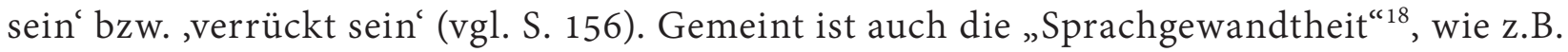
im folgenden Satz: „Sie war bereit, sich waschen zu gehen, aber ihre Füße waren noch nicht bereit, ihr zu gehorchen“ (S. 94f.). Aus dieser Perspektive entsprechen, Reeve zufolge, die zahlreichen Übersetzungen und sprachlichen Referenzen des Suahelis bei Zweig einerseits ihrem „detachment from German culture“, und andererseits „the strengh of her continuing affinity to Kenya and the language and culture she adopted there“. ${ }^{19}$

Als Kind gelingt es Vivian - im Gegensatz zu ihrem Vater - durch ihre Bereitschaft,

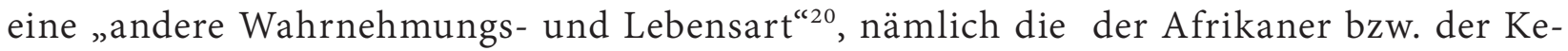
nianer, anzunehmen. So schafft sie, die Geheimnisse der lokalen Sprachen zu erkennen, sich, dank u.a. ihrem Jugendfreund Jogona und ihrem englischen Freund Kinghorn, in Kenia anzupassen, und sich somit eine neue Heimat anzueignen. Unter diesem Blickwinkel widerspricht sie Jogona unmittelbar, wenn er behauptet, dass die Nandi keine Fremden

16 Djoufack, Patrice:. „Ich habe aus meiner alten Heimatsprache übersetzt“, S. 60. In diesem Sinne ist es von der homodiegetischen Erzählerin Regina [hier Vivian] in Irgendwo in Deutschland folgendes zu lesen: „Das war nicht ich. Ich habe nur aus meiner alten Heimatsprache übersetzt. Suaheli. [...]. Ich denke nur manchmal in Suaheli. Das hilft" (Zweig, Stefanie: Irgendwo in Deutschland, S. 229).

17 Shestokat, Karin U.: Memories of Africa: Stefanie Zweig's Autobiographical Works, S. 59; „She [Vivian] speaks with the Africans in their language(s) and also imitates their mode of speaking, their playfulness and descriptveness, their flowery imaginatives and allegorical use of words, images, and metaphors. For example, there seems to be a certain ritual when asking questions although one often already knows the answers"(ebd, S. 55).

18 Leonie Marx: Konkurrierende Netzwerke im kenianischen Exil, S. 34.

19 Reeve, Jennifer: 'No common mother tongue or fatherland', S. 121.

20 Djoufack, Patrice: „Ich habe aus meiner alten Heimatsprache übersetzt“, S. 59. 
mögen, und unterstreicht dabei, dass sie keine Fremde sei (vgl. S. 181). Bemerkenswert daran ist, dass ihre eminente Integration ihr bewusst ist. Denn sie hält sich nicht mehr für eine Fremde im Exilland, sondern als Angehörige. Dies zeigt, wie sie sich nicht nur auf der Farm, also unter den Kikuyu, zu Hause fühlt, sondern in ganz Kenia. Ein weiterer Faktor, der zeigt, wie Vivian im Vergleich zu ihrem Vater in Afrika eingegliedert ist, lässt sich durch ihren Umgang mit Frauen und Männern wie auch mit Kindern ihres Alters beobachten. Vivian bedeutet viel für alle, und alle sind auch wichtig für sie. Sie lieben sie, wie sie auch alle liebt. So zeigen sie ihr beispielsweise ihre Freude, sie wiederzusehen, durch ihre Freundlichkeit, ihr Lächeln und die lokalen Lieder v.a. das Lieblingslied der Protagonistin bei ihrer Rückkehr von der Schule. Ihr wird nämlich von allen auf der Farm stets ein herzliches Willkommen bereitet:

\begin{abstract}
Vivians Heimkehr hatte sich bereits überall herumgesprochen. Die vertrauten Menschen, nach denen es sie drei Monate verlangte hatte, standen vor dem Haus, die Männer, die Frauen und Kinder. Sie stampften, johlten und klatschten und sangen das Lied vom Schakal, der einen Schuh gefressen hat und weinen muss. Vivian war gerührt. Sie hatte das Lied gern; das hatte man auf der Farm nicht vergessen. (S. 152)
\end{abstract}

Im Folgenden möchte ich darüber einen Abriss geben, wie Vivian sich, im Gegensatz zu ihrem Vater, konkret die kenianische Kultur, Sprache und damit eine neue Heimat aneignet. Durch „eine permanent geübte Mnemotechnik und Rhetorik“ ${ }^{21}$ bzw. ein ständiges Beobachten und Mitmachen, wie auch aus Neugier (vgl. S. 63), sammelt Vivian Erfahrungen ${ }^{22}$, die ihr nicht nur eine Zugehörigkeit bzw. ein Zuhause schenken, sondern auch aus ihr eine erwachsene Figur machen. Sie erlebt u.a. die traditionelle Entbindung von Mama Warimu und entdeckt zum ersten Mal, wie ein Kind auf die Welt kommt (vgl. S. 100f.). Dieses Ereignis, das eigentlich nur Erwachsene miterleben dürfen, und an dem Vivian nur dank Jogona teilnehmen darf (vgl. S. 100), bringt eine plötzliche Veränderung in ihr Leben (vgl. S. 101f.): Psychologisch hat sie ab jetzt weniger Angst als zuvor - sie fürchtet sich nicht mehr vor Blut (vgl. S. 102). Zudem entsteht eine Selbstbestätigung bei ihr. So „begriff sie, dass sie ein Geheimnis erfahren hatte, das aus Mädchen Frauen machte" (S. 101). Sie verhält sich von nun an wie eine erwachsene Frau: „Das Kind weint.“ (S. 101) teilt sie Jogona kurz nach der Entbindung in einem Gespräch mit (vgl. S. 101). Als er widerspricht, er habe nichts gehört, offenbart sie ihm triumphierend, dass Männer das Weinen des Kinds nicht sofort hören (vgl. S. 101). Darüber hinaus hat das Alter Ego Zweigs, im Vergleich zu anderen jüdischen Kinderfiguren „[...] a much more limited continuity in her family life and Jewish culture, both because the family was not strictly Orthodox and because of her [Vater's] own diffi-

\title{
$21 \mathrm{Ebd}$.
}

22 Dazu meint Leonie Marx: „Stefanies sprachlicher Umgang mit ihren afrikanischen Freunden wie auch ihr Verkehr unter den Frauen in den Hütten, wo sie mehr lernt als ihren Eltern es lieb ist, zeigt ihre enge Beziehung zu diesem Netzwerk, in das sie von Owuor souverän eingeführt wurde, dem sie auch ihre Sprachgewandtheit verdankt“" (vgl. Marx, Leonie: Konkurrierende Netzwerke im kenianischen Exil, S. 34). Darüber hinaus kennt sich Vivian auch in der lokalen Küche aus. Sie kann z.B. die lokale Speise, Maisbrei, kochen (vgl. S. 68). 
culties in adjusting to African life. ${ }^{23}$

Die Figur des Vaters wird im Roman also doppelt geprägt: Sieht er in seiner Heimatlosigkeit in der Fremde wie ,ein Kind' (vgl. u.a. S. 108; S. 126), ,traurig', ,verloren' (vgl. S. 110;

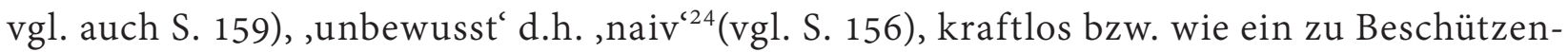
der (vgl. S. 159), wie ein Versager (vgl. S. 161), ängstlich (vgl. S. 76 und S. 159f. in der Episode zu der Figur Morenu) oder ungeduldig (vgl. z.B. S. 112) aus, so bekommt der europäische Leser Mitleid mit ihm und zeigt daher Verständnis für seine aktuelle schwierige Lage in der Fremde und fühlt seine Schmerzen und sein Leiden mit: „Die Worte machten den Tag süß. Choroni wäre zufrieden gewesen, sie immer wieder zu kosten [...], aber dem Bwana war nicht zu trauen. Er verstand sich nicht auf Gespräche. Er wusste nicht, dass die Worte immer wieder neu gesagt werden mussten“ (S. 107). Und gerade weil der Vater nichts von Dingen versteht, die um ihn passieren (vgl. S. 62) wird sein Gedächtnis als „das Gedächtnis eines Affen" (S. 107) bezeichnet. Taucht er aber als eine verständliche, sympathische, emotionelle und liebvolle Gestalt auf, so lobt ihn der Leser in seinem Umgang mit Menschen und Tieren auf der Farm (vgl. S. 112; S. 124).

Weiß die Tochter, dass der lokale Ausdruck sterben wollen nur eine Metapher für tot sein ist (vgl. S. 124f.), so ignoriert der Vater, dass einer sterben wollen kann, und dass, wenn dies vorkommt (wie im Fall der Figur von Manjala, dem kleinen Bruder Jogonas), nichts mehr hilft, auch nicht das Allheilmittel „Hustensaft“ (S. 124). Dies ist der Fall, trotzt der langen Erklärung Kimanis: „Wenn ein Mensch oder ein Tier in Afrika sterben »wollte«, so starben sie. Dann half nichts mehr. Man durfte den Tod nicht aufhalten. Alle Leute auf der Farm wussten das. Alle außer dem Bwana" (S. 68). Eine weitere Szene, wo der Vater ernsthaft unter der lokalen Kultur leidet, liegt in der Binnengeschichte zu Morenu, von dem Vivian eine Nacht träumt. Dabei hört sie, wie de Bruin ihren Vater vor dem kranken und verrückten Morenu warnt: „Hör mal, wir müssen ihn finden“ (S. 155). Der Vater fragt wieder, wen sie finden müssen (vgl. S. 156). Dabei erscheint er „wie ein Kind, das eine Frage stellt, obwohl jeder die Antwort kennt“ - außer ihm (S. 156). Während alle außer Vivian große Angst vor Morenu haben (vgl. S. 156), kann der Vater kaum nachvollziehen, wie hoch die Gefahr sei, die von Morenus Rückkehr auf die Farm ausgeht: „Ein verrückter Mann auf der Farm kannte nur noch Blut und Tod. [...]" (S. 156). So beobachtet Vivian genauer Morenu und versteht, dass sein Eintritt ins Haus mit Schuhen eine Kampfansage bedeutet (vgl. S.158). Nun macht sie sich, wie auch de Bruin, Sorgen um ihren Vater, denn der Kampf zwischen ihnen wurde schon seit langem angekündigt und es gibt kein Zurück mehr (vgl. S. 158). Und all diese traditionellen Konnotationen kennt Vivian, ihr Vater aber nicht, weshalb er wieder als jemand erscheint, der zu wenig sieht (vgl. S. 158):

Es stimmte also, was alle über Morenu sagten. Er war verrückt. Alle wussten es, nur ihr Vater nicht. Er wusste nie etwas von den Dingen, was um ihn geschahen. Er sprach nur von Dingen, die kein anderer verstand. Auch was jetzt mit Morenu geschah, würde er nicht begreifen, bis es zu spät war. (S. 159)

23 Reeve, Jennifer: 'No common mother tongue or fatherland', S. 127.

24 Dabei will er z.B. die „Memsahib“ Hanna heiraten, obwohl er nicht sicher ist, ob sie eine gute Frau für ihn sei (vgl. S. 107f.). Außerdem weiß er auch gar nichts über seinen eigenen Spitznamen, „Schießpulver“, bis de Bruin es ihm bei seinem Besuch erklärt (S. 87). 
Aber es gelingt der Tochter, mit ihrem gesparten Geld Morenu aus dem Haus zu jagen. So rettet sie nicht nur das Leben ihres Vaters, sondern auch das all der anderen. Dabei bedankt sich der Vater bei seiner Tochter und erkennt ihren Mut an (vgl. S. 161). In diesem Sinne dient die Figur des Vaters dem europäischen/deutschen Leser zumindest teilweise auch als Identifikationsfigur, da diesem (im Unterschied zu seiner Tochter) die kenianischen Gepflogenheiten oftmals fremd bleiben.

\section{2. „NO COMMON FATHERLAND“: WAS IST HEIMAT FÜR WEN?}

Wurde im vorherigen Kapitel untersucht, wie unterschiedlich die Figuren von Vater und Tochter auf der kulturellen, sprachlichen Ebene in der Fremde dargestellt werden, so interessiert mich im Folgenden eher die Heimatkonzeption bei den jeweiligen Figuren. Dabei gehe ich hauptsächlich folgenden Fragenstellungen nach: Was repräsentiert Heimat für beide Figuren? Welches sind die Hintergründe für diese Wahrnehmungsunterschiede?

Der Roman thematisiert vorwiegend das Heimatkonzept, d.h. den Heimatverlust und den Erwerb einer neuen Heimat. Zeigt die Tochter Interesse an Afrika und hält Kenia für Heimat, so zeigt der Vater kein Interesse an Afrika und bezeichnet Kenia nur als ein Aufnahmeland. Dies obwohl die Afrikaner (z.B. Jogona) ihm die Freude und den Anlass bieten, $\mathrm{zu}$ lachen und zeitweise glücklich auszusehen, was er gerade in Deutschland nicht mehr erreichen kann: „Der Bwana lachte sehr selten, aber wenn Jogona mit ihm sprach, lachte er manchmal plötzlich los, und das schmeichelte Jogona sehr" (S. 58). Als der Vater seine Tochter fragt, ob es stimme, dass sie Lehm gegessen habe und sie ihn antwortet, weshalb sie das tun sollte, reagiert er wütend und bezeichnet das Aufnahmeland als ein „Kaffernland“: „weiß der Himmel, weshalb. In diesem Kaffernland ist alles möglich“ (S. 63).

Ist die Tochter fasziniert vom fremden Land und Leben, von Landschaft und Kultur, so hält der Vater das Aufnahmeland und fast alles, was damit verbunden ist, für „verflucht“ (S. 145). Während er versucht, sich an den zweiten Vers von Mörikes Gedichts Er ists ${ }^{25} \mathrm{zu}$ erinnern, und die Tochter ihn fragt, ob er zaubere, widerspricht der Vater irritiert: „Nein, kannst du nicht einmal diesen ganzen verfluchten Zauber vergessen?“ (S. 74). In der Tat bezeichnet Afrika/Kenia für Vivian das Herz (Emotionalität), also eine emotionale Heimat, während Deutschland hingegen das Hirn (Rationalität ${ }^{26}$ beinhaltet. Insofern wird Deutschland von Vivian zwar rational als Ort ihrer Herkunft aufgefasst, diese kommt jedoch nicht der Heimat ihres Herzens gleich (vgl. u.a. S. 202):

Vivian hielt sich die Ohren zu. Immer sprach ihr Vater von Deutschland und vom Krieg. Wusste er denn nicht, dass Deutschland ein Wort war, das auf ihrer Brust wie ein Stein lag? Wusste er immer noch nicht, dass die Farm in Ol' Joro Orok ein Paradies war und es nichts Schöneres gab als die Farm? (S. 162)

25 Mörike, Eduard: Er ists. In: Werke in einem Band, hrsg. v. Herbert G. Göpfert, S. 29.

26 Deutschland wirkt hier als ein logischer und rationaler Raum, während Afrika mit Unlogik und „Irrationalität“ konnotiert wird. Göttsche, Dirk: Zwischen Exotismus und Postkolonialismus, S. 168. 
Deshalb versucht sie sogar ihren Vater von ihrer Konzeption ihrer neuen Heimat zu überzeugen, in der Hoffnung, dass er eines Tages seine Rückkehr nach Deutschland (trotz Kriegsende) aufgeben wird: „Es gibt nichts Schöneres als Ol’ Joro Orok“ (S. 162) sagt sie zu ihrem Vater und streichelt „[...] ihn wie eine Frau, die einen Mann verzaubern will“ (S. 162). Denn sie „kannte viele Zaubersprüche für Männer, die nicht mehr in ihrer eigenen Hütte leben wollten" (S. 162). Gemeint ist hier mit dem lokalen Ausdruck, nicht mehr in der Hütte leben wollen' metaphorisch ,untreu werden'. Der Vater pflegt jedoch, seine Tochter ständig daran zu erinnern, dass sie nicht aus Afrika stammt, sondern aus Deutschland (vgl. u.a. S. 65), wie es beispielsweise bei einem Gespräch zwischen Tochter, Kinghorn und Vater heißt. Während Vivian sich in Kenia zu Hause fühlt und sich einbildet, dort ewig zu bleiben, bringt Kinghorn ihr zur Erkenntnis: „Du wirst nicht immer in Afrika bleiben, kleine Memsahib“ (S. 175), und versucht dem Vater folgendes zu klären: „Es wird schwer für Vivian sein, wenn sie eines Tages von Afrika fort muss. Sie ist hier zu Hause“ (S. 175). Darauf reagiert der Vater autoritär: „Sie muss ihr Zuhause erst kennenlernen“ (S. 175).

Dabei wird dem Leser die inszenierte Nuance zwischen „zu Hause“ (Fremde) und "Zuhause“ (Heimat, d.h. Deutschland) vermittelt. Nur ungern beschreibt Vivian einmal bei einem ihrer Fragespiele ihrem Jugendfreund ihre Herkunft wie folgt: „Ich kenne ein Land, das ganz anders ist als Afrika. Es heißt Deutschland. [...] Ich komme aus Deutschland“ (S. 54). Dabei reflektiert sie über ihre alte Heimat, indem sie das Herkunftsland und das Aufnahmeland kontrastiert. Bei einem Abschiedsgespräch informiert sich Vivian bei Kinghorn, der im Gegensatz zu Vivians Vater in Kenia sehr integriert ist, über ihre europäische Heimat:

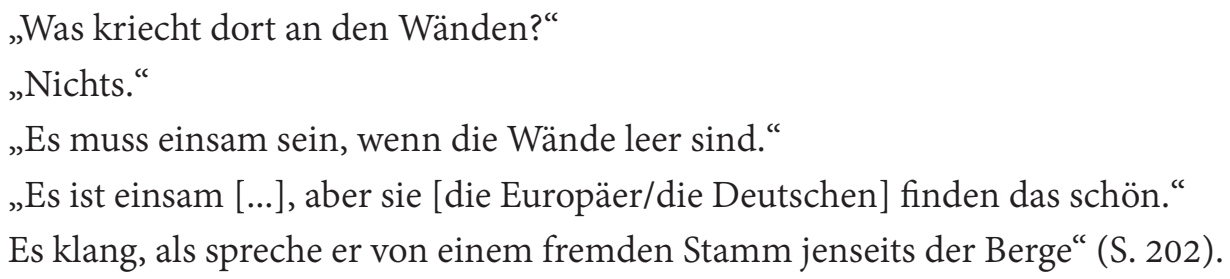

Die gebrauchte anonyme Form, die für Europa nämlich für die Europäer im zitierten Passus steht (,sie'), zeigt noch deutlich, dass Europäer/Deutsche für Vivian und v.a. für Simba in Bezug auf die Afrikaner wenig bedeuten. Stattdessen definiert sich Simba als Afrikaner (Afrika als Herzensheimat), identifiziert sich mit Vivian, die kurz vor ihrer Rückkehr aus ihrer, mère patrie' (Afrika) nach Europa steht, und zeigt Mitleid für sie. Denn für ihn und Vivian heißen Heimat Kenia/Afrika, für Vivians Vater hingegen Deutschland/ Europa:

„Was hört man denn dort, wenn man auf Regen wartet?“ [...]

„Man wartet in Europa nicht auf Regen. Sie haben es verlernt, die Erde trinken zu sehen."

Er dachte an die drei Tage seines Lebens, die er in England verloren hatte, und sein Herz litt für Vivian, die Afrika nie aus ihrem Herzen bekommen würde. (S. 202) 
Um die Protagonistin über ihre antizipierte Sehnsucht hinwegzutrösten, bestärkt sie Kinghorn darin, dass alles in Afrika auf sie warten werde, auch der Regen (vgl. S. 203). Diese implizite Einladung zu einem weiteren Wiedersehen mit ihrer afrikanischen Heimat weiß Vivian zu schätzen, die Anrede „kleine Kikuyudame“ aber lehnt sie ab und widerspricht ihrem englischen Freund: „Ich bin keine Kikuyudame [...] du hast es immer gesagt, doch es war nur ein Spiel. Nicht wahr, es war nur ein Spiel?" (S. 203). Dies ist auch ein wichtiger Hinweis dafür, dass sich die fünfjährige Protagonistin im Laufe der Handlung bzw. während des zehnjährigen Aufenthalts entwickelt habe (erwachsen geworden sei) und begreift endlich, dass sie, wenn sie eine Kikuyudame wäre, ewig in Kenia bleiben dürfe und nicht nach Deutschland mit ihrem Vater zurückkehren müssen würde. Doch beruhigt sie Kinghorn: „Nein, es war kein Spiel. [...] Es war dein Leben. Du wirst nichts vergessen“ (S. 204). Dabei verweist er auf die Kindheit der Protagonistin und vergleicht die Tochter wieder mit ihrem Vater: „Du bist wie dein Vater. Du kannst nichts vergessen“ (S. 204) und meint, Vivian werde nie ihre afrikanische Heimat vergessen. Denn Afrika sei ein Teil von ihr geworden, genauso wie Deutschland ein Teil von ihrem Vater geworden sei.

Auch Kinghorns Versprechen, dass er doch gar nicht sterben kann, bis Vivian nach Afrika zurückkäme (vgl. S. 206), beruhigt sie: „[...] Vivian lehnte sich zu ihm hinüber und berührte die Narbe über seinem rechten Auge mit dem Ziegenhaar des Medizinmanns. Überwältigt spürte sie, dass der Zauber noch einmal wirkte“ (S. 206). Besonders erscheint das bildhafte und traditionelle Ereignis mit dem Tod des alten Mannes als ein sehr großer Trost für sie, das sie für ein Ereignis hält, das nicht zu versäumen sei: „[...] Wer soll mich vor die Hütte tragen, kleine Memsahib, wenn du nicht hier bist?“ (S. 206).

Wie bereits evoziert, ist der Protagonistin nun bewusst, dass sie und ihr Vater keine gemeinsame Heimat haben. Deshalb versucht sie, mit Hilfe des afrikanischen Medizinmanns, ihrem Vater seine verlorene Heimat wiederzubringen: „Vivian dachte an ihren Vater. Nur der Muchau konnte ihm helfen, konnte ihm die verlorene Heimat wiederbringen, von der er immer sprach“ (S. 185). Dieser Versuch spielt sowohl darauf an, dass ein Äquivalent des deutschen Heimatbegriffs in anderen Sprachen fehlt, als auch auf die Tatsache, dass Heimat unwiederbringlich ist; „Sie überlegte angestrengt, wie sie das dem Muchau klarmachen sollte. Weder in Kikuyu noch in Suaheli gab es ein Wort für »Heimat«". Sie konnte dem Muchau nichts von den Träumen ihres Vaters erzählen.“ (S. 185). So gibt Vivian die Idee auf, dass ihrem Vater vom Medizinmann geholfen werden könne. Stattdessen lässt sie sich in diesem Augenblick von einem auf Ziegenhaar basierendem Ritual zum Erhalt von Freunden entführen (vgl. S. 188).

Diese Verführung zeigt auch, wieviel wichtiger für die Protagonistin Afrika ist als Deutschland. Es heißt weiter: „Sie hatte an die Nandi, an den Bwana Simba und an Jogonas Beschneidung gedacht, aber nicht an Deutschland, wohin ihr Vater wollte“ (S. 185). Immerhin stellt sich Vivian vor, „[d]er Medizinmann würde den Großvater nach Afrika holen und dem Vater eine Heimat geben“ (vgl. S. 62), dann würde „nun alles gut werden“ (S. 62). Dabei ignoriert die Heldin, dass nicht nur seine Familie ihrem Vater in Afrika fehlt, sondern auch sein Land, wie der allwissende Erzähler es weiterführt:

Der Muchau würde ihren Großvater nach Afrika holen und ihren Vater wieder 
glücklich machen. Er würde ihm die »Heimat« wiedergeben. Der Muchau würde wissen, was Heimat war, auch wenn Vivian es nicht wusste. Vivians Beine schmerzten, als sie aufstand, aber sie fühlte sich groß und stark. (S. 59f.)

Tatsächlich taucht Vivians Vater in Kenia fast immer traurig auf; dies trotz seines relativ jungen Alters (vgl. S. 62). Er denkt immer nur an seine verlorene Heimat. Das Land, wo er gerade lebt, dessen Leute und Kultur, interessieren ihn weniger als seine Rückkehr nach Deutschland. Deshalb weißt er auch nur wenig über das Leben auf der Farm, im Gegensatz zu seiner Tochter, die sich gut eingelebt hat: „[...] Ihr Vater wusste so vieles nicht. Er hatte keine Ahnung von den Dingen, die das Leben schön machten. Er sprach immer nur von Deutschland und wie gut dort alles war. Dabei war Ol' Joro Orok der schönste Ort auf der Welt" (S. 62). So wird er, wie bereits observiert, an mehreren Stellen im Roman als ein Dummkopf gegenüber seiner vernünftigeren Tochter dargestellt: „Kessu war das meistgebrauchte Wort auf der Farm. Es war ein Zauber. Vivian hatte das schon lange begriffen. Ihr Vater nicht" (S. 62).

Das Heimatdenken erscheint in diesem Sinne, wie es etwa bereits von vielen Heimattheoretikern unterstrichen wurde, als eine Reflexion, die den Reflektierenden zu einer blinden und naiven Person macht, die zum Gefangenen der eigenen Gedanken wird. Vivians Vater als Heimatreflektierender kann sich nicht in seinem Aufnahmeland, Kenia, wohl fühlen; mehr noch: er weiß kein anderes Land außer seiner verlorenen Heimat zu schätzen (vgl. u.a. S. 107). Gibt es für die Protagonistin kein Zuhause mehr außer ihrer afrikanischen Heimat, so entspricht Deutschland für den Vater der einzigen Heimat - wie es in einem Gespräch zwischen beiden Figuren zu lesen ist: „Wir sind arm. Wir haben alles verloren“ (S. 65). Dabei hieße, ,seine Heimat verlieren', implizit auch, alles zu verlieren. Doch für Vivian als eine naive und noch nicht erwachsene Figur ist es „[...] ein immer wieder neues Rätsel, dass ihr Vater so viel von Dingen sprach, die er verloren hatte“ (S. 65): „Aber die Farm haben wir nicht verloren." (S. 65).

So werden Tochter- und Vaterfigur im Laufe der Handlung als Gegenfiguren beschrieben. Vivian genießt die Zeit des zehnjährigen Aufenthalts in Afrika, fühlt sich dort zu Hause und schafft dort Heimat, während der Vater diese Zeit nur als eine Migrationszeit genauer als eine Exilzeit betrachtet und demgemäß Kenia nur für ein Exilland hält: „Vivian [...] genierte sich ein wenig für ihn und seine Torheit, und doch bedauerte sie ihn. [...] In diesem Land, das ihm so fremd war, war sie die klügere von beiden. Eines Tages würde er nach Deutschland zurückkehren und seinem Kind die Heimat wiedergeben“ (S. 78)

Diese kreative Antinomie der Autorin zwischen Tochter und Vater in ihrer jeweiligen Heimatvorstellung wirkt dann jedes Mal stärker, wenn die Rede von der Rückkehr nach Deutschland ist. In der Tat erreicht der Plot seinen Höhepunkt ab dem 13. Kapitel (S. 166f.): Hier erfährt der Leser nämlich viele Veränderungen in der Diegese (Jogonas Verschwinden vor dem Beschneidungsritual, Ankündigung des Kriegsendes und der Rückkehr der Familie nach Europa). Während es Vivian schwer fällt, Afrika zu verlassen (vgl. u.a. S. 174), freut sich der Vater riesig, endlich Afrika zu verlassen (vgl. S. 190). So entsteht eine Rollenverschiebung beider Figuren bei ihrem Heimatdenken. Erstens erscheint die Tochter von nun an als der unglückliche Charakter in der Fremde, im Unterschied zu dem glücklichsten Vater: 
Seitdem der Krieg zu Ende war, hatte der Vater überhaupt keinen Sinn mehr für die Dinge, die wirklich wichtig waren. Ob de Bruin von der nächsten Ernte sprach oder Choroni eine Kuh krankmeldete, der Vater sagte stets »Bald sind wir zu Hause«. Sein Gesicht wirkte dabei, als hätte er endlich die Zauberformel gefunden, nach der er so lange gesucht hatte. (S. 189)

Das erste Mal hatte der Vater auf dem Weg zur Flachsfabrik von zu Hause gesprochen. Vivian hatte zunächst nicht begriffen [...] Erst an seinem Gelächter, das auf einmal ganz anders geklungen hatte als sonst, hatte sie gemerkt, dass er von Deutschland sprach, wenn er zu Hause sagte. (S. 190)

Zweitens erscheint Vivian hier als eine dumme Figur, die ihrem Vater gegenüber Dinge zu spät versteht. So redet sie von dem kommenden Regen auf der Farm, während der Vater von der Rückkehr nach Deutschland spricht (vgl. S. 190): „Es wird unser letzter Regen sein“ (S. 190). Eigentlich begreift Vivian zu spät, „[...] dass die Rede von der Heimkehr nach Deutschland kein neues Spiel war, wie sie zuerst gehofft und dann so sehr gewünscht hatte. Es war nie ein Spiel gewesen, wenn ihr Vater davon gesprochen hatte, dass er nicht in Afrika bleiben wollte [...]" (S. 190). Außerdem bereitet sich die Tochter darauf vor, ihrem Vater „entgegenzutreten“ (S. 200) und dies zur Zeit des täglichen Melkens, ohne zu ahnen, dass Zeit für die Heimkehr gekommen war und daher keine Zeit mehr für das Melken blieb.

Genauso wie der Aufbruch nach Kenia geschieht, verläuft nun auch die Rückkehr nach Deutschland: kein Happy-end für die Protagonistin. Jedes Mal, wenn die Rede von Deutschland ist, strahlt Vivians Vaters Gesicht: „Die Trauer war aus den Augen ihres Vater verschwunden, wenn er von Deutschland sprach. Die Lieder, die er nun sang, klangen anders als zuvor. [...]“ (S. 190). Dabei nimmt die Tochter nur schwer Abschied von Afrika und anderen Figuren wie z.B. von Bwana Simba, der ihr lehrt, „[m]an soll nicht bei Tage Abschied nehmen“ (S. 207): „[...] Vivian spürte, dass sie die Tränen nicht mehr lange zurückhalten konnte. „Kwaheri“ [...] „Kwaheri, Bwana Simba.““ (S. 207). Mit einer starken Emotionalität erlebt der Leser, den afrikanischen Zauber des Abschieds, der zwischen den beiden Figuren entsteht:

Kwaheri, meine kleine Memsahib. Komm gut heim von deiner Safari, rief er und ritt fort, ohne sich umzudrehen. Es war ein alter Zauber, sich beim Abschied nicht in die Augen zu blicken. Wer einen Freund wieder sehen wollte, musste in der Stunde der Trennung (beim Abschied) Stärke beweisen. (S. 207)

Inzwischen genießt auch der Vater die sanfte Landschaft auf der Farm, selbst wenn er es nicht zugibt/zugeben will. Kenia wird irgendwie auch seine ,Heimat': „Der Bwana stand an der wuchernden Hecke mit den lila Kletterblumen und dunkelgrünen Blättern“ (S. 103); aber das ist nie sein Heimatland geworden. Da Exil „verbrannt, ausgegrenzt, gezwungen zu sein, ein anderes Land zu finden, um dort eine neue Existenz aufzubauen [...]“ heißt, bleibt dabei offen, ob es zu einer Akkulturation oder zu einer Rückkehr, eventuell zu keiner 
dieser beiden Möglichkeiten kommt. “27 Im Fall der Figur des Vaters löst das Exil eben keine Akkulturation in der Fremde aus, vielmehr bleibt dieser von Anfang bis Ende statisch in seinem ethnozentrischen Denken. Von diesem Befund ausgehend möchte ich nun der zweiten Fragestellung nachgehen: Welches sind die inszenierten Gründe für die herrschende Dichotomie zwischen Vater- und Tochterfigur bei ihrer jeweiligen Heimatkonstruktion?

Dass es kein gemeinsames Vaterland für Tochter und Vater im Roman gibt, ist kein Zufall. Dies liegt eher im Altersunterschied zwischen beiden Figuren begründet. Die erwachsene Figur des Vaters ${ }^{28}$ nimmt das Exil und das damit assoziierte Schicksal schmerzhaft wahr: „Wir gehen jetzt in ein anderes Land [...]. Alles, was wir geliebt haben, lassen wir zurück. Unsere Heimat haben wir verloren." (S. 58). Der noch naiven fünfjährigen Figur der Tochter, die sich kaum an Deutschland erinnern kann (vgl. S. 59), hingegen fällt schwer das Gesamte zu begreifen: „Auch das hatte Vivian nicht verstanden. Man verlor Taschentücher und Bleistifte, aber wer verlor schon eine Heimat?" (S. 58f.)

So zeigt sich die Tochter bereit zu einem neuen Leben in der Fremde, während der Vater sich die ganze Exilzeit nach seiner deutschen Heimat sehnt und von seiner Rückkehr nach dieser träumt. Demnach versteht der Vater fast nichts auf der Farm und fühlt sich fremd, unsicher in Kenia - im Gegensatz zu seiner ehemaligen Heimat, Deutschland, wo er die Geborgenheit genießt (vgl. S. 106). Dabei kann die Tochter kaum die Vorsicht und die Angst des Vaters auf der Farm nachvollziehen. Deshalb spielt sie damit, wie es in einem Dialog zwischen Vater und Tochter zu lesen ist: „Morenu trägt immer ein Messer bei sich“ [...]. „Das hast du mir schon erzählt.“ [...]. (S. 76) Doch trotz dieser spontanen Entgegnung des Vaters setzt die Tochter ihr Spiel fort: Er [Morenu] hat gesagt: „Eines Tages schieße ich alle Weißen tot." (S. 76) Meine Vermutung hier ist, dass die Binnengeschichte über Morenu den Vater an den Antisemitismus in Deutschland erinnert, während die Tochter daran viel Spaß findet. Für ihn haben die von Morenu mit „alle Weißen“ bezeichneten Opfer eine doppelte Bedeutung: Alle Deutschen auf der Farm und im weiteren Sinne alle Juden in Deutschland. Der Vater erfährt Fremdheit schließlich doppelt, da er sich nicht auf Afrika einlässt: Einerseits ist er in Deutschland fremd, wo er wegen seiner jüdischen Herkunft ausgeschlossen und verfolgt wird; andererseits ist er fremd in Afrika, wo er jede Integration ablehnt und sich somit ebenfalls ausschließen lässt. Dabei ignoriert der Vater im Unterschied zu seiner Tochter, dass zwischen Afrika und Europa nicht nur eine geographische Distanz, sondern auch eine ganze Welt liegt. Die Tochter nimmt z.B. den Kontrast zwischen der Farm Ol' Joro Orok und der Stadt Nakuru zur Kenntnis (z.B. während ihrer Schulzeit), wo sich ein neues Leben insbesondere in Verboten ausdrückte:

Sobald Vivian so weit war, die Dinge zu begreifen, merkte sie, dass alles verboten war, was sie gern getan hatte. Zwischen der Farm Ol' Joro Orok und der Schule in Nakuru lagen nicht hundert Meilen, sondern eine ganze Welt. Es war verboten, sich anzuziehen, wie man wollte. [...]. (S. 136)

27 Marx, Leonie: Konkurrierende Netzwerke im kenianischen Exil, S. 11.

28 Als „Territoriale[r] Mensch“ sieht er sein ganzes Leben mit seinem ursprünglichen Territorium verbunden und seine Identität im gleichen Territorium, das ihm Verhaltenssicherheit gewährt, da in ihm „Umwelt als Lebenswelt durch ihr Selbstverständnis zur Eigenwelt geworden ist“. Greverus, Ina-Maria: Der territoriale Mensch, jeweils S. 385 und S. 54. 
Die Tatsache, dass die Vaterfigur im Laufe des Romans ihrer deutschen Heimat auch im Exil und damit unter schwierigen Lebensbedingungen treu bleibt, verweist auf Brechts Vorstellung des Exils, nach der dieses (im Sinne von Vertreibung) ${ }^{29}$ sich von Auswanderung unterscheidet. Brecht zufolge bedeutet auswandern, ein freier „Entschluss des Auswanderers in ein von ihm frei gewähltes Land heraus, wo er ein neues Heim finden kann“, während Exil für ihn „ein aufgezwungenes Übersiedeln in die nächstmögliche Fremde“ darstellt. ${ }^{30}$ Unter Brechts Blickwinkel sieht der Vater der Protagonistin das Exilland, Kenia, als einen Ort, der „eine Chance zum Überleben“31 bietet (vgl. u.a. S. 145), aber keine Heimat ersetzen soll. Diese Attitüde des Vaters bezeichnet seine Resolution dazu, dass Kenia nur als Aufnahmeland bleibt und nie zum Vaterland wird; ähnlich wie bei Brecht in den Versen 8-9: „Und kein Heim, ein Exil soll das Land sein, das uns da aufnahm. “" Und dieser Entschluss bei der Vaterfigur ist stark von einer großen Hoffnung gefärbt. So betont er oft „»Bald sind wir zu Hause “" (S. 189). Zudem sorgt er dafür, dass keine räumlich „überschreitbare Grenze gezogen werden kann, zwischen der bedrohlich und gewalttätig gewordenen Heimat und einer Rettung versprechenden Fremde, welche das auch immer sei “. ${ }^{33}$ So projiziert Vivians Vater eine Rückkehr in seine deutsche Heimat, sobald dort der Krieg aufhört (siehe u.a. Zitat oben: S. 189), wie es im folgenden Gespräch zwischen ihm und seiner Tochter zu lesen ist:

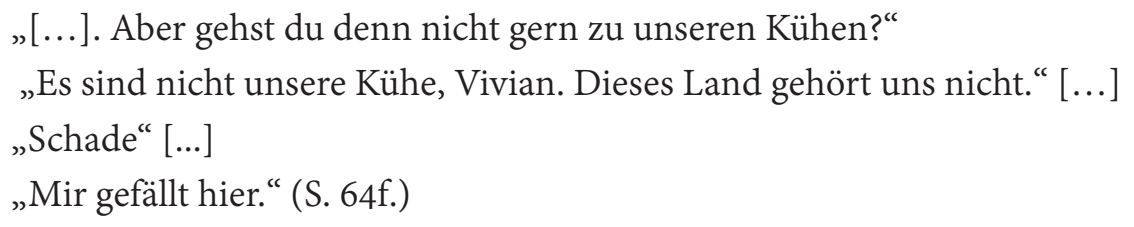

Unter diesen Umständen, wo der Vater in Bezug zu seinem deutschen Heimatbegriff seinen ethnozentrischen Charakter offenlegt, scheitert jeder Versuch der Tochter, ihrem Vater durch ihre teilweise neugierigen und für sie witzigen Fragen Freude zu machen und damit ihm einen neuen Heimatraum anzubieten ${ }^{34}$ : „Vivian sagte ihrem Lächeln Jambo. Es war schön, wie leicht ihr Vater in Fallen ging. Von nun an würde sie ihn immer Bwana nennen. Der Gedanke machte sie so gut gelaunt, dass sie ihrem Vater eine Freude machen wollte." (S. 64). Ohne überhaupt zu ahnen, wie schmerzvoll für den Vater es war, von Deutschland zu erzählen, bittet sie ihn, ihr von seinem Land zu erzählen (vgl. S. 64). Dabei betont der Vater seine finanzielle Lage in der Fremde, da die Tochter alles für ein Spiel zu halten scheint: „Wir sind arm. Wir haben alles verloren. [...] Also ein für alle Mal [...] wir sind arm

29 Brecht, Bertolt: Über die Bezeichnung Emigranten. In: Jan Knopf (Hrsg.): Lebenskunst in finsteren Zeiten, S. 404: „[... ] Vertriebene sind wir, Verbannte", Vers 7.

30 Djoufack, Patrice:. „Ich habe aus meiner alten Heimatsprache übersetzt“, S. 47; vgl. dazu auch Brecht, Bertold: Ebd.

31 Djoufack, Patrice: „Ich habe aus meiner alten Heimatsprache übersetzt“, S. 47.

32 Brecht, Bertolt: Über die Bezeichnung Emigranten. In: Jan Knopf (Hrsg.): Lebenskunst in finsteren Zeiten, S. 404.

33 Djoufack, Patrice: „Ich habe aus meiner alten Heimatsprache übersetzt“, S. 47

34 Wobei Deutschland, die bedrohliche Heimat nichts mehr als „Nicht-Ort“ in der Tat bedeuten soll. „Nicht-Orte“ sind nach Augé Orte der Anonymität, die denjenigen, die sich an ihnen aufhalten, keine Identifikation erlauben. Augé, Marc: Orte und Nicht-Orte, S. 92f. Vgl. dazu auch: Schlink, Bernhard: Heimat als Utopie, S. 32. 
wie die Kirchenmäuse“ (S. 65). Dem starken Bild aus der christlichen Religion bzw. dem Bild der „Kirchenmäuse“ bedient sich hier der Vater, um nochmal seiner Tochter und auch implizit dem Leser seine finanzielle Schwierigkeiten in Afrika zu vermitteln.

Ein weiterer Hintergrund dafür, dass es bei dem Vater zu keiner Akkulturation in der Fremde kommt, liegt am „finanzielle[n] Handicap mit seinen psychischen Konsequenzen“. ${ }^{35}$ Nachdem der Vater mit dem Exil alles (Heimat, Beruf und Besitz) verloren hat, muss er ein neues/fremdes Leben in Afrika anfangen. Diese schwierigen neuen Lebensbedingungen werden dem Leser durch einen Dialog zwischen Tochter und Vater vermittelt:

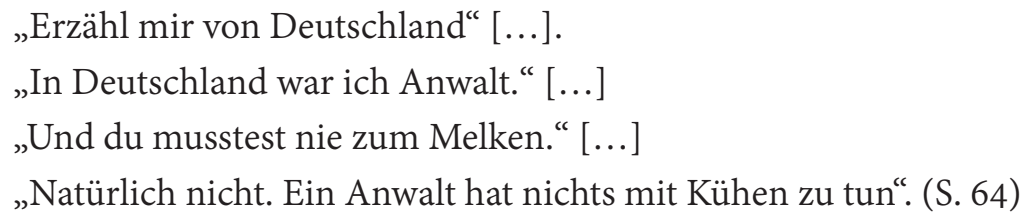

Da der Vater mit seinem neuen sozialen Rang und finanziellen Zustand in der Fremde nicht zufrieden ist, schafft er sich dort auch keinen Heimatersatz. Er kann z.B. die Schulgebühren für seine Tochter mit seinem geringen Gehalt eines Melkers, wovon er und seine Tochter leben müssen, erst bezahlen, nachdem diese gesenkt wurden: „Seit ein paar Wochen gab es in Kenia die allgemeine Schulpflicht für Europäer. Vivians Vater war glücklich, denn für Leute mit wenig Geld wurden die sehr hohen Schulgebühren gesenkt." (S. 132) Dieser Punkt, der wiederum auf die historischen und politisch-ideologischen Hintergründe hinweist, führt dazu, dass Tochter und Vater keine gemeinsame Heimat im Roman teilen. In der Tat erleben die deutsch-jüdischen Flüchtlinge in Kenia ständig Diktatur und Diskriminierung durch die britischen Kolonialbehörden (vgl. u.a. S. 131f.), was die Vaterfigur nicht länger erdulden kann. So repräsentiert Kenia für den Vater „keine Alternative zu Deutschland. “ ${ }^{36}$ Deshalb entscheidet er sich nach Kriegsende, mit seiner Tochter nach Deutschland zurückzukehren. Für ihn bleibt Deutschland der ideale Ort, der ihm „Heim, Haus, Schutz ${ }^{\text {“37 }}$ gewährleistet. In dem Sinne bedeutet der Heimatbegriff „ein besonderes emotionales Verhältnis insbesondere zu einer kleinräumlichen Lebenswelt mit allen ihren Elementen [...], das zur personalen und sozialen Integration führt“ “ ${ }^{38}$

\section{LITERATURVERZEICHNIS PRIMÄRLITERATUR}

ZWEIG, Stefanie. Ein Mund voll Erde. Frankfurt/Main: Fischer, 2003 [erste Auflage 1980].

ZWEIG, Stefanie. Vivian. Frankfurt/Main: Fischer, 2003 [erste Auflage 2001].

35 Marx, Leonie: Konkurrierende Netzwerke im kenianischen Exil, S. 28.

36 Djoufack, Patrice: „Ich habe aus meiner alten Heimatsprache übersetzt“, S. 56.

37 Türcke, Christoph: Heimat, S. 7.

38 Gauger, Jörg-Dieter: Heimat - Tradition - Geschichtsbewusstsein - Bemerkungen zu einem vermuteten Zusammenhang. 
ZWEIG, Stefanie. N in Afrika. München: Heyne, 2007 [erste Auflage 1995].

\section{Sekundärliteratur}

AKTUELL KULTUR: „Bestseller-Autorin Stefanie Zweig gestorben“. In: https://www.dw.com/de/ bestseller-autorin-stefanie-zweig-gestorben/a-17594522, veröffentlicht am 27.04.2014, abgerufen am 13.07.2020.

AUGÉ, Marc. Orte und Nicht-Orte. Vorüberlegungen zu einer Ethnologie der Einsamkeit. Übersetzt von Michael Bischoff. Frankfurt/Main: S. Fischer 1994. Übersetzt von Michael Bischoff. Frankfurt/ Main: S. Fischer, 1994.

BRECHT, Bertolt. Über die Bezeichnung Emigranten. In: Jan Knopf (Hrsg.): Lebenskunst in finsteren Zeiten. Biografie. München. Carl Hanser, 2012.

DJOUFACK, Patrice. „Ich habe aus meiner alten Heimatsprache übersetzt. Suaheli.“ Interkulturalität und Übersetzung bei Stefanie Zweig. In: Leo Kreutzer u. David Simo (Hrsg.): Weltengarten. Hannover: Revonnah 2004, S. 45 - 64.

EMRE, Merle. Grenz(über)gänge. Kindheit in deutsch-türkischer Migrationsliteratur. Würzburg: Königshausen \& Neumann, 2014 (Interkulturelle Moderne. Bd. 5).

GAUGER, Jörg-Dieter. Heimat - Tradition - Geschichtsbewusstsein - Bemerkungen zu einem vermuteten Zusammenhang. In: Heimat - Tradition - Geschichtsbewusstsein. 1986, (Studien zur politischen Bildung, Bd. 11), S. 9-44.

GÖTTSCHE, Dirk. Zwischen Exotismus und Postkolonialismus. Der Afrikadiskurs in der deutschsprachigen Gegenwartsliteratur. In: M. Moustapha Diallo u. ders. (Hrsg.): Interkulturelle Texturen: Afrika und Deutschland im Reflexionsmedium der Literatur. Bielefeld: Aisthesis, 2003, S. 161244.

GREVERUS, Ina-Maria. Der territoriale Mensch. Ein literaturanthropologischer Versuch zum Heimatphänomen. Frankfurt/Main: Athenäum, 1972.

MARX, Leonie. Konkurrierende Netzwerke im kenianischen Exil: Zwischenpositionen der Familie Stefanie Zweigs. In: Helga Schreckenberger (Hrsg.): Networks of Refugees from Nazi Germany: Continuities, Reorientations, and Collaborations in Exile. Leiden/Boston: Brill, 2016 (Amsterdamer Beiträge zur neueren Germanistik, Bd. 87), S. 11-37.

MÖRIKE, Eduard. Er ists. In: Herbert G. Göpfert (Hrsg.): Werke in einem Band. München, Wien. Carl Hanser, 1977 [4.Auflage].

REEVE, Jennifer. 'No common mother tongue or fatherland': Jewish Refugee Children in British 
Kenya. In: Simone Gigliotti u. Monica Tempian (Hrsg.): The Young Victims of the Nazi Regime. Migration, the Holocaust and Postwar Displacement. Bloomsbury/London/Oxford/New York/New Dehli u. Sydney: Bloomsbury academic, 2016, S. 113-131.

SCHESTOKAT, Karin U. Memories of Africa: Stefanie Zweig's Autobiographical Works. In: Journal of the Australasian Universities Language and Literature Association 108, 2007, S. 51-63.

SCHLINK, Bernhard. Heimat als Utopie. Frankfurt/Main: Suhrkamp, 2000.

TÜRCKE, Christoph. Heimat. Eine Rehabilitierung. Springe/Hannover: Zu Klampen, 2006. 